\title{
To oints
}

$\sqrt{0}$

\section{Treatment of chronic lateral ankle instability using the Broström-Gould procedure in athletes: long-term results}

\author{
ADRIANO RUSSO ${ }^{1}$, PAOLO GIACCHÈ ${ }^{1}$, ENRICO MARCANTONI ${ }^{1}$, ANNALISA ARRIGHI ${ }^{2}$, \\ LUIGI MOLFETTA ${ }^{2}$
}

1 Orthopaedic Institute of Florence (IFCA), Villa Ulivella e Glicini, Florence, Italy

2 University of Genoa, School of Medical and Pharmaceutical Sciences, Genoa, Italy

\begin{abstract}
Purpose: this study was conducted to evaluate longterm results following treatment of chronic lateral ankle instability using the Broström-Gould technique in athletes.

Methods: eighteen athletes involved in competitive sports at different levels, who suffered from chronic lateral ankle instability, underwent Broström-Gould ligamentoplasty between 2000 and 2005. The results of the surgery were evaluated using the American Orthopaedic Foot and Ankle Society (AOFAS) scale.

Results: the results at 10-15 years of follow-up were excellent in $94.5 \%$ of these cases and good in the remaining 5.5\%. An increase of 31.2 points in the AOFAS scale score was recorded at follow-up (with the score rising to 98.8, from 67.6 preoperatively). All the athletes returned to their respective sports at the same level as prior to the surgery. Imaging at longterm follow-up showed no signs of arthritic degeneration.

Conclusions: the results of this study show that the Broström-Gould technique is an effective procedure for the treatment of chronic lateral ankle instability in the athlete, giving excellent long-term results.

Level of evidence: therapeutic case series, level IV.
\end{abstract}

Keywords: ankle, instability, reconstruction, ligament, Broström-Gould.

\section{Introduction}

The stability of the ankle joint is provided by three

\section{Corresponding Author:}

Adriano Russo, MD

Via Fieschi 400, 19131 Cadimare (SP), Italy

E-mail: studiomedico.russo@libero.it groups of ligaments: the lateral ligaments, the tibiofibular syndesmosis and the medial ligaments. The lateral ligaments comprise the anterior talofibular ligament, the calcaneofibular ligament and the posterior talofibular ligament. The calcaneofibular ligament is an extra-capsular cord-like structure that stabilizes both the tibiotarsal (ankle) joint and the talocalcaneal (subtalar) joint. The posterior talofibular ligament is the strongest of the lateral ligaments $(1,2)$. A structure that is not, in itself, a mechanical stabilizer of the ankle but is important in the execution of the surgical technique in question is the extensor retinaculum, whose anatomy and function have been described in detail by Marconetto and Parino (3). Microscopically, its structure appears richly innervated with free endings and Pacinian corpuscles, which seems to indicate a more proprioceptive than mechanical function. Indeed, the extensor retinaculum, by activating the eversor muscles, exerts a positive effect on the stability of the ankle and also of the subtalar joint.

Conservative treatment of ankle ligament injuries does not always result in anatomical and functional healing of the injury itself or resolve the instability, and it may, in time, lead to secondary osteoarthritis. Moreover, the ankle is influenced by the morphology of the foot; cavovalgus is the morphotype that most predisposes to the risk of recurrent sprains in supination. The clinical picture may therefore be one of instability or laxity alone, or instead the two conditions may be associated. There exist numerous surgical techniques (over 50 described in the literature) (4) for treating chronic instability due to laxity caused by external ankle ligament injury. The most widely used include techniques that use the peroneus brevis tendon, such as the Watson-Jones, Evans, Larsen, Chrisman-Snook and Pisani (associated with subtalar stabilization) techniques; methods involving the use of a drawbridge-like fibular periosteal flap, such as the 
Chiappara-Rettagliata and Priano (with arthroscopic assistance) ones; and methods involving direct repair of the ligaments, such as: the Broström and Broström technique modified by Gould.

The present study evaluates long-term results of the Broström-Gould technique used for treatment of chronic lateral ankle instability in athletes.

\section{Methods}

We conducted a retrospective study of a consecutive series of athletes affected by chronic lateral ankle instability, who were treated surgically using the Broström-Gould technique between 2000 and 2005.

\section{Participants}

The criteria for inclusion in the study were the following: chronic lateral ankle instability with failure of previous conservative treatment (pharmacological treatment, braces, physical therapy and active exercise programs) and participation in competitive sports. Diagnosis of lateral ankle instability was initially based on the clinical history (self-reported episodes of instability associated with pain during normal walking, particularly on uneven ground, with progressive limitation of sports activities). Clinical examination consisted of the anterior drawer test at $20^{\circ}$ of plantar flexion, to evaluate the anterior talofibular ligament, and the talar tilt test, involving application of inversion force, to evaluate the calcaneofibular ligament. The findings were compared with the healthy side and confirmed on stress radiographs performed bilaterally using the Telos stress device. Complete rupture of the anterior talofibular ligament with intact calcaneofibular ligament and posterior talofibular ligament were also documented on ultrasound and magnetic resonance imaging.

Patients with osteochondral lesions and/or lesions of the tibiofibular syndesmosis were excluded from the study.

\section{Intervention}

The patient was placed supine under spinal anesthesia and a pneumatic tourniquet was placed at the root of the thigh. A linear incision was performed along the anterior margin of the distal fibula starting $5 \mathrm{~cm}$ proximal to the apex of the fibula and ending, after curving downwards and posteriorly, several $\mathrm{cm}$ before the peroneal tendon sheath. This was therefore a shorter incision than the one used in the classic technique, given that the patients being treated with this method did not present associated calcaneofibular ligament injuries. We were careful to avoid injuring the superficial peroneal nerve anteriorly, while the sural nerve, being posterior to the peroneal tendons, was located in an area that was sufficiently distant from the incision. We continued the dissection until the joint capsule was reached and simultaneously identified the inferior extensor retinaculum. We left $3 \mathrm{~mm}$ of capsular tissue inserted in the fibula. Within the joint capsule, the anterior talofibular ligament was identified as a thickening of the capsule (Fig. 1). We sutured the distal remnant of the anterior talofibular ligament directly to the fibula, anchoring it to the periosteum and suturing it over the abovementioned capsular tissue using mattress sutures (Fig. 2). Then the extensor retinaculum was isolated (Fig. 3), pulled over the suture and anchored to the fibula (Fig. 4). We completed the repair by suturing the subcutaneous layer and skin and protected the joint with a soft bandage. The day after surgery all the patients were fitted with a protective ankle brace with two valves and permitted to load the joint very lightly (i.e. to graze the floor) when using two crutches. From the fifteenth day post-surgery, walking was allowed with partial loading (progressively increasing), and a re-education program was started with flexion-extension exercises of the ankle and knee followed by exercises to strengthen the peroneal tendons and proprioception exercises. Running was resumed from the third month post-surgery and the patients gradually returned to their sporting activities as from the fourth month post-surgery, with protective taping used for a further two months.

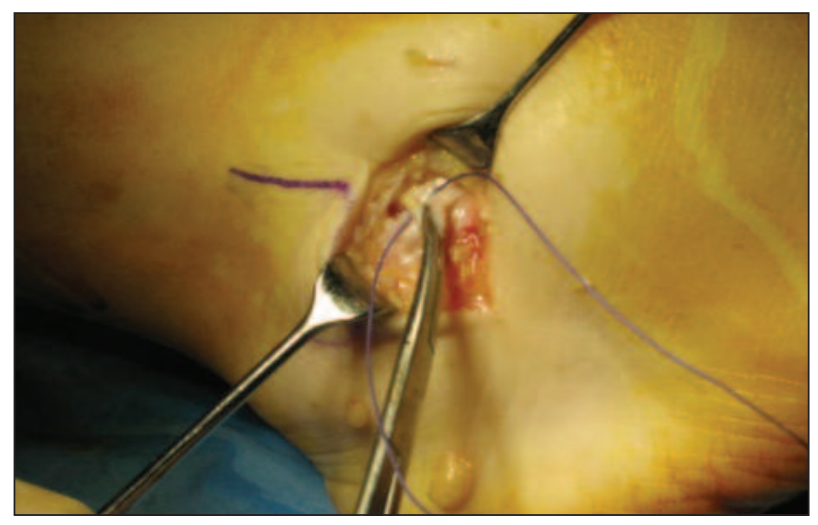

Fig. 1. The capsule and the remnant of the anterior talofibular ligament are isolated, sectioned and sutured. 


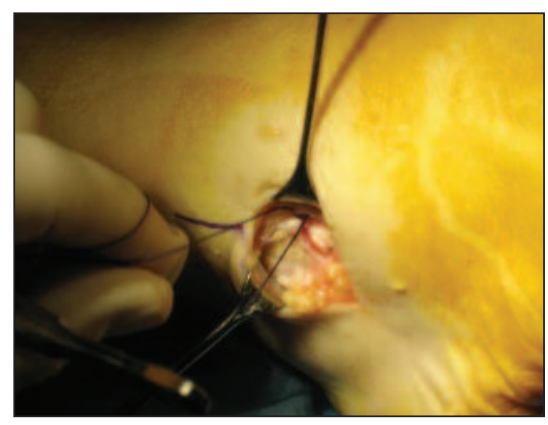

Fig. 2. The capsule and the remnant of the anterior talofibular ligament are sutured (using mattress sutures) to the fibular periosteum.

\section{Outcome measurement}

Results were evaluated using the American Orthopaedic Foot and Ankle Society (AOFAS) scale, which gives a maximum score of 100 and evaluates pain (40 points), functional status (50 points) considering functional limitation, use of supports, distance covered, ground characteristics, step alterations, sagittal movements, etc., and alignment of the foot with the ground (10 points). In addition, a podoscopic examination was performed and standard radiographs were taken.

\section{Results}

Of a total of 22 patients surgically treated between 2000 and 2005 and meeting the study inclusion criteria, 4 were lost to follow-up over the years. Therefore, the final study sample comprised 18 patients (11 males, 7 females), who were aged between 17 and 32 years (mean 21.5 years) at the time of surgery. The right side was affected in 12 patients and the left side in 6. The duration of instability ranged from a minimum of one year to a maximum of four years. All the patients practiced a sport at competitive level, specifically: soccer $(n=8)$, tennis $(n=1)$, artistic gymnastics $(n=2)$, basketball $(n=2)$, athletics $(n=1)$, dance sports $(n=1)$, and volleyball $(n=3)$. Preoperative failed treatments consisted of immobilization in a plaster cast and rehabilitation in 10 patients, while the remaining 8 patients had previously been treated with braces and then undergone rehabilitation.

All the patients were reassessed after an interval of time ranging from a minimum of 10 years to a maximum of 15 years.

No perioperative infectious, vascular or neurological complications were observed in any patient. The
AOFAS score rose from a mean of 67.6 before surgery to a mean of 98.8 at the final follow-up, corresponding to a mean increase of 31.2 points. The longterm results were excellent in $94.5 \%$ of the cases and good in the remaining $5.5 \%$. There were no unsatisfactory results in this series of patients.

In detail, the results were the following. Pain was absent in 17 patients, and occasional in one; likewise, walking was normal in 17 patients, while one had occasional difficulties, when walking on uneven surfaces. No case had presented severe lameness and gait difficulties. Ankle flexion-extension movement was complete in all cases without secondary stiffness; 2 patients showed moderate limitation of supination. With regard to the mechanical alignment of the ankle, the findings were a moderately positive anterior drawer in one case and anterior-posterior stability in the remaining cases, while all the cases showed varus-valgus stability. As for the alignment of the heel and footprint, one patient had calcaneal valgus and flatfootedness; another had calcaneal varus. These abnormalities predated the surgical treatment.

All the patients were submitted to a podoscopic examination to document support abnormalities. In 15 subjects the foot showed normal alignment of the heel and an unremarkable footprint; one patient had bilateral cavus foot, one had a mild calcaneal varus deformity ipsilateral to the lesion, and another had a moderate calcaneal valgus deformity with first-degree flat foot ipsilateral to the lesion.

Radiographic examination showed no signs of arthritic degeneration in any of the cases treated.

All the patients returned to their various sporting activities, practicing them at the same level as prior to the surgery. Furthermore, the surgery did not affect any patient's eventual decision to stop practicing their sport; indeed, this decision depended exclusively on 
personal choice or on the passing of time, and was independent of the surgical treatment received.

\section{Discussion}

The present study showed excellent long-term results in athletes affected by chronic ankle instability treated with the Broström-Gould ligamentoplasty technique. In accordance with the literature, we obtained extremely satisfactory results with this surgical technique, which proved to be simple and rapid, and free from intra- and post-operative complications. It did not necessitate the sacrifice of tendons that is required by other techniques, and the post-operative course was relatively pain free and easily tolerated by the patients. The Authors attached considerable importance to the preoperative radiographic examination performed using the Telos stress device, since today this is, objectively, probably the most appropriate technique for determining talar translation and talar tilt. While aware of the reproducibility limits of this examination, in agreement with most Authors, we take $9 \mathrm{~mm}$ of anterior translation and $15^{\circ}$ of talar tilt as limits of normality (5). We feel that performing an arthroscopy step before proceeding with the ligament reconstruction is helpful in cases with associated and documented intra-articular pathology, as it allows this problem to be properly addressed in the course of the same procedure (6-8). The excellent stability obtained with the Broström-Gould method in these treated cases, followed up after an interval of 10-15 years, their rapid recovery of joint function, and their return to sport are all factors that convince us to continue using this surgical technique and encourage us to use it in injuries of the calcaneofibular ligament, as suggested by the inventor of the technique. Bell et al. (9) reported long-term results obtained when using this method to repair the calcaneofibular ligament in a sizeable active duty military population. The results in these patients at 26.3 years of follow-up were found to be excellent or good in $91 \%$ of the cases. Corte-Real et al. (10) obtained good results with a low incidence of major and minor complications. Nery et al. (11) stated that the arthroscopic BroströmGould-assisted technique could be a viable alternative to the gold-standard Broström-Gould procedure, as it allows the treatment of intra-articular lesions. Wainright et al. (12) recently reported improved ankle joint kinematics in unstable ankles after modified BroströmGould repair, with a significant decrease in anterior translation and internal rotation of the talus. The clinical evaluation criteria applicable to joints repaired using the
Broström-Gould technique are not yet standardized. Buerer et al. (13), comparing four different scores, found a high rate of satisfaction in terms of articular mobility, albeit in the presence of a disparity of long-term outcomes. Cadaveric studies have shown greater mechanical stability obtained with the modified Broström technique as opposed to Watson-Jones and Chrisman-Snook reconstructions $(14,15)$, especially in competitive athletes, which encourages us to apply this method in this specific category of athletes in particular.

\section{References}

1. Gould JS. Operative foot surgery. Philadelphia, WB Saunders, 1994.

2. Bordmann DL, Liu SH. Contribution of the anterolateral joint capsule to the mechanical stability of the ankle. Clin Orthop Relat Res. 1997;(341):224-232.

3. Marconetto M, Parino E. Il retinacolo inferiore degli estensori, anatomia funzionale, utilizzo nelle plastiche legamentose. Chirurgia del piede. 2003;2:36-41.

4. Giannini S, Ceccarelli F, De Benedictis M, et al. Lesioni capsulo legamentose croniche della tibiotarsica. In: Progressi in chirurgia del piede [Congress Proceedings]. Il piede nello sport. 1997;7-139.

5. Karlsson J, Lansinger O. Lateral instability of the ankle joint. Clin Orthop Relat Res. 1992;(276):253-261.

6. Cannon LB, Slater HK. The role of ankle arthroscopy and surgical approach in lateral ankle ligament repair. Foot Ankle Surg. 2005;11:1-4.

7. Salvi AE, Metelli GP, Pazzaglia UE. Artroscopia di caviglia nello sportivo. Minerva Ortopedica Traumatologica. 2004;55: 257-263.

8. Messer TM, Cummins CA, Ahn J, et al. Outcome of the modified Broström procedure for chronic lateral ankle instability using suture anchors. Foot Ankle Int. 2000;21:996-1003.

9. Bell SJ, Walthour CS, Provencher MT, et al. Chronic lateral ankle instability: the Broström procedure. Operative Techniques in Sports Medicine. 2005;13:176-182.

10. Corte-Real NM, Moreira RM. Arthroscopic repair of chronic lateral ankle instability. Foot Ankle Int. 2009;30:213-217.

11. Nery C, Raduan F, Del Buono A, et al. Arthroscopic-assisted Broström-Gould for chronic ankle instability: a long-term follow-up. Am J Sports Med. 2011;39:2381-2388.

12. Wainright WB, Spritzer CE, Lee JY, et al. The effect of modified Broström-Gould repair for lateral ankle instability on in vivo tibiotalar kinematics. Am J Sports Med. 2012;40:20992104.

13. Buerer Y, Winkler M, Burn A, et al. Evaluation of a modified Broström-Gould procedure for treatment of chronic lateral ankle instability: a retrospective study with critical analysis of outcome scoring. Foot Ankle Surg. 2013;19:36-41.

14. Liu SH, Baker CL. Comparison of lateral ankle ligamentous reconstruction procedures. Am J Sports Med. 1994;22:313-317.

15. Hoy GA, Henderson IJ. Results of Watson-Jones ankle reconstruction for instability. The influence of articular damage. J Bone Joint Surg Br. 1994;76:610-613. 